\title{
Die Mentalismus-Frage in der Nervenheilkunde
}

Das nervenheilkundliche Verständnis psychischer und neurologischer Gesundheitsstörungen steckt nach wie vor in dem Dilemma, das man seit alters her das LeibSeele-Problem nennt: Es ist bislang nicht möglich, zwischen dem subjektiven Erleben - der mentalen Sphäre - und dem organismischen Geschehen - etwa der neuronalen Verkörperung des Mentalen - eine lückenlose Brücke zu schlagen. Da selbst in der Kognitionswissenschaft nicht zweifelsfrei klar ist, was genau wir unter dem Mentalen verstehen sollten, ist die Mentalismusfrage weiterhin aktuell: Wie ist das Verhältnis von Seelischem und Körperlichem am besten zu verstehen? Und was ist das überhaupt: Mentales?

In diesem Heft kommen Positionen zur Sprache, die sich nicht mit der bloßen Gleichsetzung („Identitätstheorie“) von mentalem Geschehen und Gehirnprozessen zufriedengeben. Der Geist, so die meisten beteiligten Autoren, beschränkt sich nicht auf das Gehirn. Denn zumindest im Fall des Menschen handelt es sich um einen verkörperten, im Kontext zu verstehenden, grundsätzlich aktiven, auf adaptives Handeln zielenden Geist. Für diese grob umrissene Auffassung wird aus theoretischer, neurowissenschaftlicher und klinischer Perspektive argumentiert.

Aus theoretischer Sicht argumentiert Henrik Walter gegen den immer noch weit verbreiteten passiven Internalismus: Wir können die Inhaltlichkeit des Geistes nur dann richtig verstehen, wenn Kontext und Geschichte in der Form eines aktiven Externalismus mit einbezogen werden. Markus Pawelzik versucht auf der gleichen Linie zu zeigen, dass der menschliche Geist nur als Vehikel der kulturellen Praxis angemessen zu verstehen ist: Wir assimilieren die Alltagspsychologie unserer Kultur, um uns selbst so zu verstehen, wie auch andere uns verstehen können.
Aus neurowissenschaftlicher Sicht ist Einiges über die neuronalen Systeme des Mental-Machens und Geist-Verstehens bekannt. So etwa liegen der Fähigkeit, die mentalen Zustände anderer zu erkennen und ihr Verhalten darauf basierend zu begreifen („theory of mind“) spezifische Systeme zugrunde, die von Wladimir Tantchik et al. dargestellt werden. Ein weiterer interessanter Ansatz in diesem Zusammenhang ist der Versuch, Gedanken direkt aus gemessenen Hirnsignalen auszulesen. John-Dylan Haynes referiert den Stand der entsprechenden empirischen Bemühungen und seine Limitationen einschließlich daraus resultierender moralischer Fragen.

Aus klinischer Sicht ist die zunehmend populäre „mentalisierungsbasierte Psychotherapie" für unser Thema von Belang, die von Svenja Taubner und Kollegen in ihrer aktuellen Form dargestellt wird. Der Mentalisierungsansatz betont ein wesentliches Ingredienz jeder Art von interpersoneller Psychotherapie: das Verstehen von Verhalten als motiviertem, durch intentionale mentale Zustände angetriebenem Handeln. In diesem Zusammenhang freuen wir uns, Peter Fonagy, dem maßgeblichen Begründer des Mentalisierungsansatzes, für ein Interview gewonnen zu haben, in dem er seinen aktuellen Vorstellungen über die Rolle des Mentalen Ausdruck verleiht.

Wir hoffen, dass dieses Spektrum unterschiedlicher Ansätze zur Mentalismusfrage die Vielfalt existierender Ansätze deutlich macht und unsere Leser dazu anregt, über die Relevanz und Natur des Mentalen vor dem Hintergrund ihres eigenen Tätigkeitsbereichs neu nachzudenken.

\section{H. Walter, Berlin}

\section{Pawelzik, Münster}

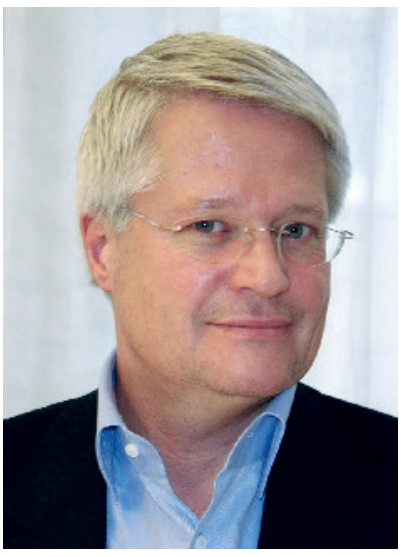

Dr. Markus R. Pawelzik, EOS-Klinik für Psychotherapie, Münster

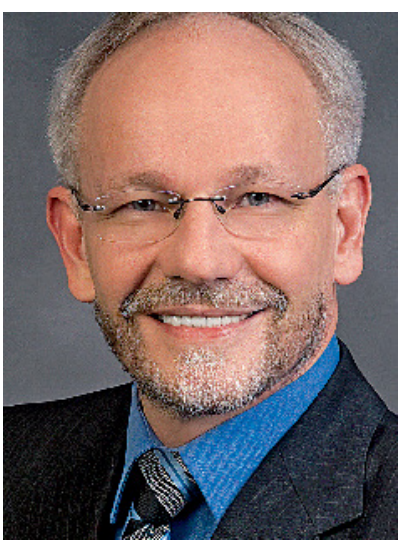

Prof. Dr. Dr. Henrik Walter, Klinik für Psychiatrie und Psychotherapie, Charité Universitätsmedizin Berlin, Campus Mitte 